grupos auxiliando na pesquisa de novas cultivares e busca de espécies silvestres requeridas como base genética para programas de melhoramento da cultura.

\section{LITERATURA CITADA}

ARIYO, O.J. Genetic diversity in West African okra (Abelmoschus caillei) (A. chev.) Stels Multivariate analysis of morphological and agronomic characteristics. Genetic Resources and Crop Evolution, v. 40, p. 25-32, 1993.

BAUM, B.R.; GUPTA, P.K. Numerical taxonomy and genome analysis. In: JAUHAR, P.P., ed. Methods of genome analysis in plants. North Dakota: CRC Press, 1996. p. 345-356.

BISHT, I.S.; PATEL, D.P.; NAHAJAN, R.K. Classification of genetic diversity in Abelmoschus tuberculatus germplasm collection using morphometric data. Annual Applied Biology, v. 130, p. 325-335, 1997.

BOHAC, J.R.; AUSTIN, D.F.; JONES, A. Discovery of wild tetraploid sweetpotatoes. Economical Botany, v. 47, p. 193-201, 1993. BRETTING, P.K.; WIDRLECHENER, M.P. Genetic markers and horticultural germplasm management. HortScience, v. 30, n. 7, p. 13491355, 1995.
BROWN, A.H.D.; CLEGG, M.T. Isozyme assessment of plant genetic resources. In: BATTAZI, M.C.; SCANDALIUS, J.G.; WHITT, G.S., eds. Isozymes: Current topics in biological and medical research. New York: A.R. Liss, 1983. p. 285-295.

CHARRIER, A. Genetic Resources of Abelmoschus (okra). Rome: IBPGR, 1984. 64 p. CRAWFORD, D.J. 1990. Plant molecular systematics: macromolecular approaches. New York: Wiley, 1990. 388 p.

CRUZ, C.D. Programa Genes: aplicativo computacional em genética e estatística. Viçosa: Editora UFV, 1997. 394 p.

CRUZ, C.D.; REGAZZI, A.J. Modelos biométricos aplicados ao melhoramento genético. Viçosa: UFV - Imprensa Universitária, 1994. 390 p.

CRUZ, C.D. Aplicação de algumas técnicas multivariadas no melhoramento de plantas. Piracicaba: USP/ESALQ, 1990. 188 p. (Tese doutorado)

HAMON, S.; VAN SLOTEN, D.H Characterization and evaluation of okra. In: BROWN, A.H.D.; FRANKEL, O; MARSHALL, D.R. ; WILLIAMS, J.T., eds. The use of plant genetic resources. Cambridge: Cambridge University Press, 1989. p. 173-196.
JOLLIFFER, I.T. Discarding variables in a principal component analysis, II. Real data. Applied Statistics, v. 22, p. 21-31, 1973.

MARTINELLO, G.E.; ABBOUD, A.C.S.; LEAL, N.R. Análise em componentes principais e de agrupamentos aplicada a características morfológicas e agronômicas em quiabeiro. Horticultura Brasileira, v. 14, n. 2, p. 200-203, 1996.

MORAIS, O.P. Análise multivariada da divergência genética dos progenitores, índices de seleção e seleção combinada numa população de arroz oriunda de intercruzamentos usando macho-esterilidade. Viçosa: UFV-Imprensa Universitária, 1992. 251 p. (Tese doutorado).

PEREIRA, A.V. Utilização de análise multivariada na caracterização de germoplasma de mandioca (Manihot esculenta Crantz). Piracicaba: USP/ESALQ, 1989. 108 p. (Tese doutorado).

SIEMONSMA, J.S. West-African okra morphological and cytogenetical indications for the existence of a natural amphidiploid of Abelmoschus esculentus (L.) Moench and A. manihot (L.) Medikus. Euphytica, v. 31, n. 1, p. 241-252, 1982

VIANA, A.E.S.; SEDIYAMA, T.; CECON, P.R.; LOPES. S.C.; SEDIYAMA, M.A.N.; Estimativas de tamanho de parcela em experimentos com mandioca. Horticultura Brasileira, Brasília, v. 20, n. 1, p. 58-63, março 2.002.

\title{
Estimativas de tamanho de parcela em experimentos com mandioca.
}

\section{Anselmo Eloy S. Viana'; Tocio Sediyama²; Paulo Roberto Cecon²; Sandro C. Lopes ${ }^{1}$; Maria Aparecida N. Sediyama $^{3}$}

${ }^{1}$ UESB, C. Postal 95, 45.100-000, Vitória da Conquista-BA; ${ }^{2}$ UFV, 36.571-000 Viçosa-MG ${ }^{3}$ EPAMIG, C. Postal 216, 36.571-000

Viçosa-MG, E-mail: marians@mail.ufv.br.

\section{RESUMO}

Com o objetivo de estimar o tamanho da parcela em experimentos com mandioca, foi realizado um ensaio, no Campus da Universidade Estadual do Sudoeste da Bahia, em Vitória da Conquista. As estimativas de tamanho da parcela foram obtidas em ensaio de uniformidade, usando-se a cultivar Cramuquém. Foram empregados os métodos da máxima curvatura, da máxima curvatura modificado e da comparação de variâncias. A estimativa do tamanho da parcela variou com a metodologia empregada e com a característica analisada. O método da máxima curvatura modificado foi aquele que permitiu a obtenção de estimativas mais adequadas. Por esse método, e considerando-se que a parcela ideal deve possibilitar a avaliação eficiente de todas as características analisadas neste experimento, o tamanho adequado de parcela encontrado foi de $26,59 \mathrm{~m}^{2}$ (44 plantas).

Palavras-chave: Manihot esculenta Crantz, coeficiente de variação, método da máxima curvatura, experimentação.

\section{ABSTRACT}

Estimates of plot sizes in experiments with cassava.

To estimate the plot size in experiments with cassava, an experiment, using the cultivar "Cramuquém" was conducted at the Campus of the "Universidade Estadual do Sudoeste da Bahia" in Vitória da Conquista, Brazil. The maximum curvature method, the modified method for maximum curvature, the method for comparison among variances were used. The estimate of the plot size changed with the applied methodology and the analyzed characteristic. The modified method for maximum curvature was the one which allowed for the obtainment of more adequate estimates. By applying this methodology and considering that the ideal plot would make possible an efficient evaluation of all analyzed characteristics, the adequate size of the plot was found to be $26.59 \mathrm{~m}^{2}$ (44 plants).
Keywords: Manihot esculenta Crantz, coefficient of variation, method for maximum curvature, experimentation.

\section{(Aceito para publicação em 5 de dezembro de 2.001)}

A mandioca (Manihot esculenta Crantz), planta pertencente à família Euphorbiaceae, é originária da América tropical, provavelmente do Nordes- te do Brasil (Dominguez et al., 1982). É cultivada em países da África, Ásia, Oceania e América Latina, onde suas raízes representam importante fonte ali- mentícia, rica em calorias e carboidratos, para aproximadamente 600 milhões de pessoas (Roca et al., 1991). 
Tabela 1. Área de cada parcela, número de parcelas, número de unidades básicas (ub) e número de plantas, no ensaio de uniformidade com mandioca, variedade Cramuquém, Vitória da Conquista, UESB, 1998.

\begin{tabular}{lcccc}
\hline $\begin{array}{c}\text { Tamanho de } \\
\text { parcela }^{*}\end{array}$ & Área $\left(\mathbf{m}^{\mathbf{2}}\right)$ & No de parcelas & $\mathbf{N}^{\circ}$ de ub & $\mathbf{N}^{\circ}$ de plantas \\
\hline $\mathrm{R}$ & 518,4 & 3 & 108 & 864 \\
$\mathrm{BI}$ & 172,8 & 9 & 36 & 288 \\
$\mathrm{P}$ & 57,6 & 27 & 12 & 96 \\
$\mathrm{SP}$ & 28,8 & 54 & 6 & 48 \\
$\mathrm{SSP}$ & 14,4 & 108 & 3 & 24 \\
SSSP & 4,8 & 324 & 1 & 8 \\
\hline
\end{tabular}

* Tamanho de parcela: $\mathrm{R}=$ repetições; $\mathrm{Bl}=$ blocos; $\mathrm{P}$ = parcelas; $\mathrm{SP}=$ subparcelas;

SSP = subsubparcelas e, SSSP = subsubsubparcelas.

No Brasil, segundo maior produtor de mandioca do mundo (FAO, 2000), trabalhos de pesquisa em campo com essa cultura são feitos em várias localidades, abrangendo ambientes bastante diversos. Muitas vezes a heterogeneidade das condições locais tem levado à obtenção de erros experimentais de grandes magnitudes, o que dificulta a comprovação estatística de diferenças entre os tratamentos avaliados.

Além das condições ambientais, é importante observar, para a cultura da mandioca, a variação existente no material de plantio, o que leva, segundo Lozano et al. (1977), à desigualdade de vigor e de produção por planta, entre plantas provenientes de uma mesma variedade. Essa desigualdade pode ser maior em regiões de baixa pluviosidade e em solos que apresentam limitações quanto à fertilidade, já que, nessas condições, as plantas de mandioca seriam, inicialmente, mais dependentes das reservas existentes no material de plantio.

A adoção de um tamanho de parcela adequado é uma das maneiras de reduzir o erro experimental. Esse tamanho ótimo da parcela é muitas vezes recomendado por meio de estudos empíricos feitos para uma região ou cultura específica. Porém, do ponto de vista estatístico, essa prática não é a melhor, já que o tamanho ótimo da parcela depende da heterogeneidade do local experimental (Lin et al., 1996).

O tamanho e a forma das parcelas não podem ser generalizados, pois variam com o solo e com a cultura. A determinação dos mesmos deve ser feita para cada cultura e cada local em que ocorram condições climáticas e de solo diferentes das que já tenham sido determinadas (Oliveira \& Estefanel, 1995). O tamanho da parcela influencia diretamente a precisão e o valor dos dados experimentais obtidos. Além da precisão estatística, vários outros aspectos são importantes para determinar o tamanho ideal da parcela, como presença ou ausência de bordadura, tipo de cultura, número de tratamentos, nível de tecnologia empregada no cultivo e disponibilidade de área e de recursos financeiros (Bueno \& Gomes, 1983).

O objetivo deste trabalho foi estimar o tamanho adequado da parcela, para experimentos com mandioca.

\section{MATERIAL E MÉTODOS}

O ensaio foi conduzido na Área Experimental do Campus da Universidade Estadual do Sudoeste da Bahia, em Vitória da Conquista. As médias das temperaturas máxima e mínima do ar são, respectivamente, de $25,3^{\circ} \mathrm{C}$ e de $16,1^{\circ} \mathrm{C}$. A precipitação média anual é de $733,9 \mathrm{~mm}$, sendo o maior nível encontrado de novembro a março. O solo da área experimental foi classificado como Latossolo Amarelo Álico A moderado, relevo plano. A análise química do solo revelou $\mathrm{pH}$ em água: 4,6; $\mathrm{P}: 1,0 \mathrm{mg} / \mathrm{dm}^{3}$; $\mathrm{K}^{+}: 0,2 \mathrm{cmol}_{\mathrm{c}} / \mathrm{dm}^{3} ; \mathrm{Al}^{3}: 0,4 \mathrm{cmol}_{\mathrm{c}} / \mathrm{dm}^{3}$; $\mathrm{Ca}^{2}: 1,3 \mathrm{cmol}_{\mathrm{c}} / \mathrm{dm}^{3} ; \mathrm{Mg}^{2}: 0,8 \mathrm{cmol}_{\mathrm{c}} / \mathrm{dm}^{3}$; $\mathrm{H}^{+}+\mathrm{Al}^{3+} 3,4 \mathrm{cmol} / \mathrm{dm}^{3}$; S.B. $\mathrm{cmol} / \mathrm{dm}^{3}$ : 2,3; m: 15,0\%; V: 40,0\%; C.T.C. Total: $5,7 \mathrm{cmol}_{\mathrm{c}} / \mathrm{dm}^{3}$.

Utilizou-se a cultivar de mandioca conhecida regionalmente como Cramuquém, cujas plantas apresentam hastes marrom-claras, sem ramificação, com broto terminal verde e pecíolo vermelho. As raízes tuberosas apresentam película suberosa de cor marrom-escura, rugoso, com córtex creme e polpa branca, com presença de pedúnculo e ausência de cintas. Essa cultivar é utilizada regionalmente, tanto para produção de farinha e extração de amido, como para consumo das raízes na alimentação humana.

O solo foi arado e gradeado e os sulcos, espaçados de um metro, foram abertos com trator. As manivas utilizadas no plantio foram obtidas de plantas sadias, com idade aproximada de 18 meses e plantadas logo após a coleta, distribuídas a cada $60 \mathrm{~cm}$ dentro de cada sulco. Foram utilizadas manivas padronizadas, provenientes do terço médio da planta, com $20 \mathrm{~cm}$ de comprimento e aproximadamente dois centímetros de diâmetro e com cinco a sete nós. O corte da maniva foi reto, em ambas as extremidades, sem usar apoio nesse procedimento.

O experimento foi plantado, sem calagem e adubação, em dezembro de 1995, formado de 27 fileiras, espaçadas de $1 \mathrm{~m}$, com 96 plantas em cada, numa área total de $1.555,2 \mathrm{~m}^{2}$, com replantio aos trinta dias após a brotação. Os tratos culturais foram restritos às capinas e ao controle de formigas.

$\mathrm{Na}$ colheita, feita em outubro de 1997, as plantas foram agrupadas em conjuntos com oito unidades denominados unidades básicas (ub), de cuja combinação foram formados os diferentes tamanhos de parcela analisados, todos com forma retangular, denominados repetições $(\mathrm{R})$ blocos $(\mathrm{Bl})$, parcelas $(\mathrm{P})$, subparcelas (SP), subsubparcelas (SSP) e subsubsubparcelas (SSSP) (Tabela 1).

Foram avaliados a porcentagem de matéria seca e amido em raízes tuberosas, 
Tabela 2. Estimativas de coeficientes de variação (\%), em função do tamanho de parcela em unidades básicas (nub), para as características rendimento de raízes tuberosas (R), peso da parte aérea (PPA), índice de colheita (IC), porcentagem de matéria seca (MS), porcentagem de amido (A) e altura da planta de mandioca (AP), Vitória da Conquista, UESB, 1998.

\begin{tabular}{rrrrrrr}
\hline $\boldsymbol{n u}^{\mathbf{1 /}}$ & $\mathbf{R}$ & PPA & IC & MS & A & AP \\
\hline 108 & 0,98 & 0,91 & 0,82 & 0,08 & 0,09 & 0,16 \\
36 & 1,55 & 1,60 & 1,30 & 0,17 & 0,20 & 0,42 \\
12 & 3,50 & 3,68 & 2,68 & 0,40 & 0,47 & 0,89 \\
6 & 5,89 & 6,25 & 4,11 & 0,78 & 0,92 & 1,70 \\
3 & 10,68 & 12,79 & 6,98 & 1,43 & 1,68 & 4,48 \\
1 & 27,14 & 31,47 & 16,99 & 3,93 & 4,63 & 12,38 \\
\hline
\end{tabular}

${ }^{1 /} n u b=8$ plantas.

Tabela 3. Estimativas das variâncias reduzidas, em unidades básicas ( $u b$ ), para índice de colheita (IC), porcentagem de matéria seca (MS), peso da parte aérea (PPA), produção de raízes tuberosas (R) e altura de plantas de mandioca (AP), nos diferentes tamanhos de parcela, Vitória da Conquista, UESB, 1998.

\begin{tabular}{cccccc}
\hline $\boldsymbol{n u b}$ & IC & MS & PPA & R & AP \\
\hline 108 & $0,0015 \mathrm{~b}$ & $0,0603 \mathrm{c}$ & $0,6271 \mathrm{c}$ & $0,4886 \mathrm{c}$ & $0,0009 \mathrm{c}$ \\
36 & $0,0013 \mathrm{~b}$ & $0,0989 \mathrm{c}$ & $0,6463 \mathrm{c}$ & $0,4094 \mathrm{c}$ & $0,0020 \mathrm{c}$ \\
12 & $0,0018 \mathrm{~b}$ & $0,1832 \mathrm{c}$ & $1,1392 \mathrm{c}$ & $0,6969 \mathrm{c}$ & $0,0030 \mathrm{c}$ \\
6 & $0,0021 \mathrm{~b}$ & $0,3467 \mathrm{c}$ & $1,6406 \mathrm{c}$ & $0,9848 \mathrm{c}$ & $0,0055 \mathrm{c}$ \\
3 & $0,0030 \mathrm{~b}$ & $0,5805 \mathrm{~b}$ & $3,4397 \mathrm{~b}$ & $1,6185 \mathrm{~b}$ & $0,0190 \mathrm{~b}$ \\
1 & $0,0060 \mathrm{a}$ & $1,4628 \mathrm{a}$ & $6,9430 \mathrm{a}$ & $3,4852 \mathrm{a}$ & $0,0485 \mathrm{a}$ \\
\hline
\end{tabular}

Numa mesma coluna, valores seguidos de mesma letra não diferem entre si, pelo teste de Bartlett, a 5\% de probabilidade.

conforme metodologia proposta por Grossmann \& Freitas (1950); altura de plantas, peso da parte aérea; peso de raízes tuberosas e índice de colheita.

A análise estatística foi feita de acordo com o critério de classificação hierárquica, simulando um experimento em parcelas subsubsubdivididas (Vallejo \& Mendoza, 1992) (Tabela 3) e as variâncias para cada tamanho de parcela, foram reduzidas em relação a uma subsubsubparcela (Hatheway \& Williams, 1958), em ordem hierárquica. Para determinação do tamanho ótimo de parcela, foram utilizados o método da máxima curvatura, o método da máxima curvatura modificado e o método da comparação de variâncias.

Pelo método da máxima curvatura, um experimento de uniformidade ou determinada área é colhido, em unidades básicas, as quais são combinadas para formar parcelas experimentais de vários tamanhos. Obtidos os coeficientes de variação, para cada tamanho de parcela, esses são representados graficamente contra o tamanho de cada parcela avaliada. O tamanho ótimo da parcela é determinado visualmente, correspondendo ao ponto de máxima curvatura (Federer, 1955). Entretanto, neste trabalho, optou-se por utilização de programa de computador (Microsoft Excel), na elaboração dos gráficos, unindo-se os pontos com segmento de reta, conforme procedimento adotado por Ortiz (1995). O aperfeiçoamento do método da máxima curvatura, chamado de Método da Máxima Curvatura Modificado, foi desenvolvido por Lessman \& Atkins (1963), que estabeleceram uma função do tipo $\mathrm{Y}=\mathrm{a} / \mathrm{x}^{\mathrm{b}}$, para explicar a relação entre coeficiente de variação (CV) e tamanho da parcela, permitindo que o ponto que corresponde ao tamanho ótimo da parcela fosse determinado algebricamente, dando maior precisão aos resultados obtidos.

Neste trabalho, usou-se a função $C V=a X^{\bar{b}}$ em que o valor da abcissa, no ponto de curvatura máxima, é dado pela seguinte fórmula (Chaves,1985), deduzida a partir de Meier \& Lessman (1971):

$$
X M C=\left[\frac{a^{2} b^{2}(2 b-1)}{(b-2)}\right]^{\frac{1}{(2-2 b)}}
$$

sendo: $\mathrm{X}_{\mathrm{MC}}=$ valor da abcissa correspondente ao ponto de máxima curvatura; $\mathrm{a}=$ constante da regressão; e, b = coeficiente de regressão.

Outro procedimento utilizado na determinação do tamanho ótimo de parcelas experimentais é a comparação de variâncias. Nesse método (Vallejo \& Mendonza, 1992; Ortiz, 1995), inicialmente as variâncias são reduzidas para uma unidade básica, dividindo-se a variância de cada parcela pelo número de unidades básicas correspondentes. Em seguida, são aplicados consecutivos testes de Bartllet, para homogeneidade de variâncias (Steel \& Torrie, 1980), excluindo-se, em cada teste, a parcela de menor tamanho que apresente variância estatisticamente diferente. Quando um grupo de variâncias homogêneas é obtido, escolhe-se o menor tamanho de parcela, dentro desse grupo, como sendo o tamanho ótimo de parcela.

\section{RESULTADOS E DISCUSSÃO}

Os coeficientes de variação apresentaram valores entre $0,08 \%$ e $31,47 \%$, que, além de inversamente proporcio- 


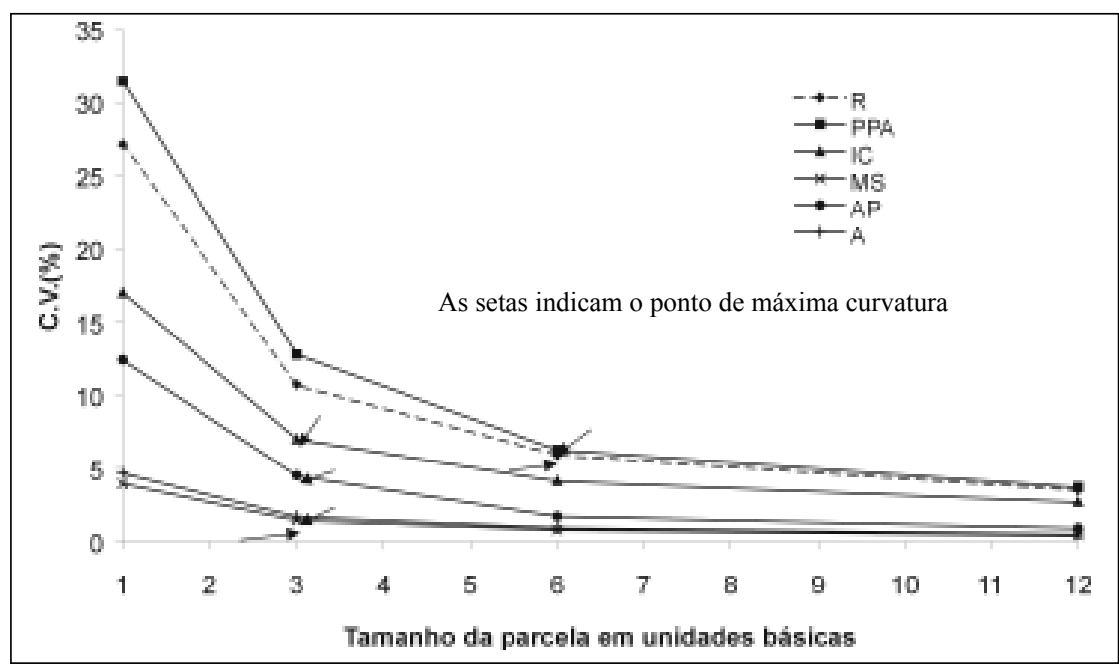

Figura 1. Relação entre coeficiente de variação e tamanho de parcela, para as características: rendimento de raízes tuberosas (R), peso da parte aérea (PPA), índice de colheita (IC), porcentagem de matéria seca (MS), porcentagem de amido (A) e altura da planta de mandioca (AP), Vitória da Conquista, UESB,1998.

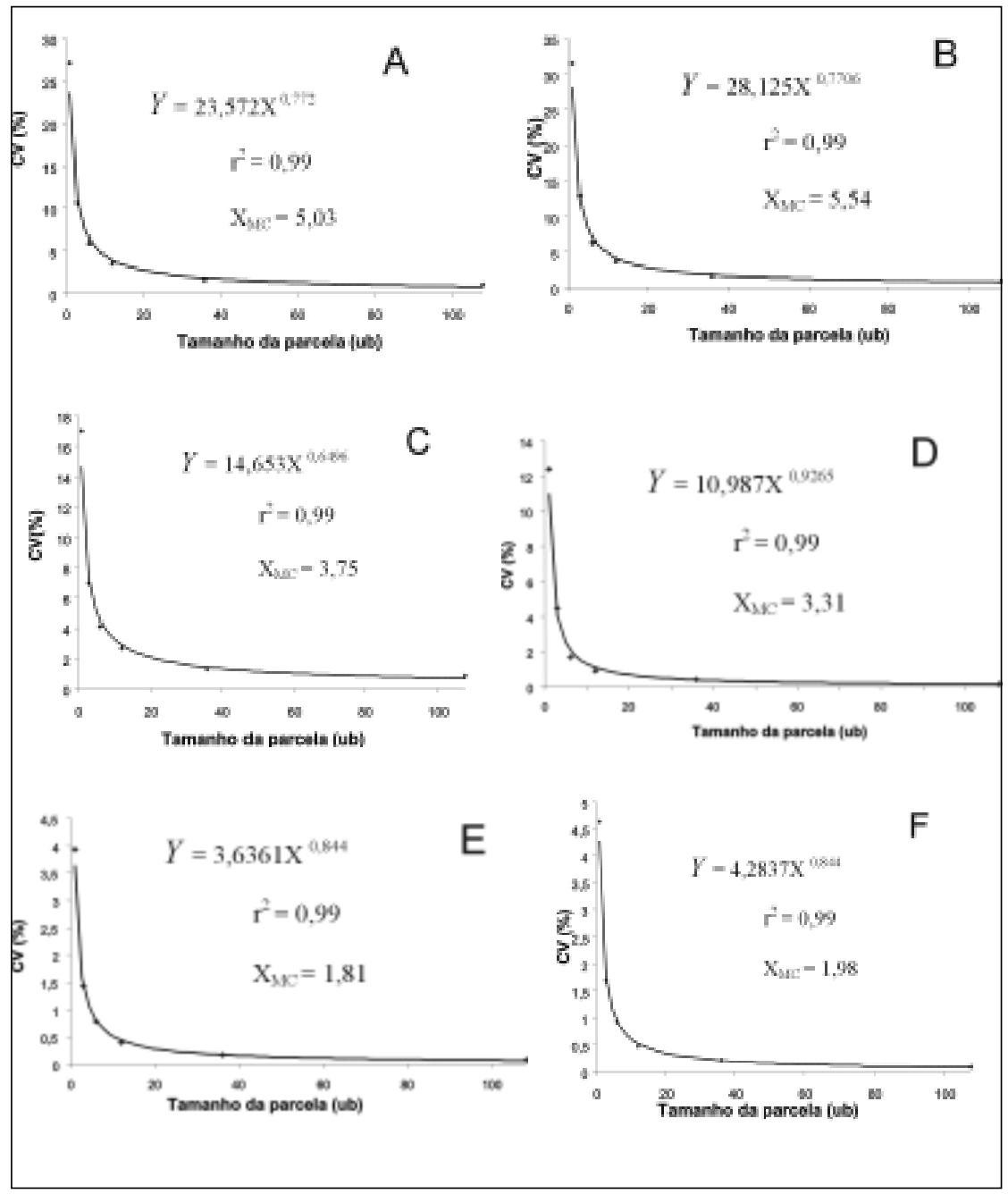

Figura 2. Relação entre coeficiente de variação $(\mathrm{CV})$ e tamanho da parcela, e valor da abcissa no ponto de máxima curvatura $\left(\mathrm{X}_{\mathrm{MC}}\right)$ para as características produção de raízes $(\mathrm{A})$, peso da parte aérea (B), índice de colheita (C), altura de plantas de mandioca (D), porcentagem de matéria seca (E) e porcentagem de amido (F), Vitória da Conquista, UESB, 1998. nais ao tamanho da parcela, mostraram comportamento diferenciado de acordo com a característica, sendo maiores para o peso da parte aérea e menores para a porcentagem de matéria seca em raízes tuberosas (Tabela 2).

Pelo método da máxima curvatura, o melhor tamanho de parcela, para rendimento de raízes tuberosas e peso da parte aérea, foi 48 plantas ou $28,8 \mathrm{~m}^{2}$ (seis unidades básicas); e para altura de plantas, índice de colheita, porcentagem de matéria seca e porcentagem de amido, em raízes tuberosas, o tamanho adequado de parcela foi 24 plantas ou $14,4 \mathrm{~m}^{2}$ (três unidades básicas) (Figura 1). Embora de fácil aplicação, este método não considera a dependência do ponto de máxima curvatura da escala usada na construção do gráfico e do tamanho da menor unidade básica adotada (Federer, 1955).

Foi observada neste trabalho a dependência da escala na determinação do ponto de máxima curvatura. Na tentativa de minimizar esse problema, na Figura 1, onde o tamanho de parcela foi determinado, usou-se uma menor escala e representou-se apenas a região de curvatura mais acentuada, compreendida entre 1 e 12 unidades básicas. Outra consideração que pode ser feita, sobre essa metodologia de determinação de tamanho de parcela, diz respeito à maneira como a curva é traçada. De acordo com a descrição de Federer (1955), após os pontos serem plotados em gráfico, uma curva, feita à mão livre, é traçada e o ponto de máxima curvatura é determinado visualmente. Esse procedimento foi adotado por Vallejo \& Mendoza (1992), que inclusive, encontraram o tamanho ótimo de parcela, no intervalo entre dois pontos. Entretanto, Ortiz (1995) optou por ligar os pontos com segmentos de reta, determinando como melhor tamanho de parcela a unidade básica correspondente à curvatura máxima. Este último procedimento foi adotado no presente trabalho.

Pelo método da máxima curvatura modificado (Figura 2) encontrou-se como tamanho ótimo de parcela: 5,03 unidades básicas (40 plantas ou $24 \mathrm{~m}^{2}$ ) para produção de raízes tuberosas; 5,54 unidades básicas (44 plantas ou 26,59 $\mathrm{m}^{2}$ ) para peso da parte aérea; 3,75 unidades básicas ( 30 plantas ou $18 \mathrm{~m}^{2}$ ) para 
índice de colheita; 3,31 unidades básicas (26 plantas ou $15,9 \mathrm{~m}^{2}$ ) para altura de plantas; 1,81 unidades básicas (14 plantas ou $8,69 \mathrm{~m}^{2}$ ) para porcentagem de matéria seca e 1,98 unidades básicas (16 plantas ou $9,5 \mathrm{~m}^{2}$ ) para avaliação da porcentagem de amido. O método da máxima curvatura modificado, embora utilize o mesmo princípio do método da máxima curvatura, fornece resultados mais precisos do que esse, por estabelecer uma equação de regressão para explicar a relação existente entre os coeficientes de variação e os respectivos tamanhos de parcela. O uso da equação de regressão permitiu, ainda, que fossem feitas estimativas de tamanho de parcela, nos intervalos entre as unidades básicas predeterminadas, nesse caso com elevada precisão, uma vez que os valores de $\mathrm{r}^{2}$ obtidos variaram de 0,98 a 0,99 .

Pelo teste de Bartllet (Tabela 3), observa-se que as variâncias reduzidas foram maiores em parcelas com uma unidade básica. Para índice de colheita, os demais tamanhos de parcela apresentaram variâncias iguais, considerando-se a parcela formada por três unidades básicas (24 plantas ou 14,4 $\mathrm{m}^{2}$ ) como a mais indicada, já que o uso de parcelas maiores não reduzirá a variância. Para as demais características avaliadas, as parcelas formadas por $6 ; 12 ; 36$ e 144 unidades básicas, apresentaram variâncias inferiores às das parcelas com uma e três unidades básicas e iguais entre si.

Os métodos utilizados neste trabalho para determinação do tamanho ótimo de parcelas experimentais têm a desvantagem de não levar em consideração os custos. Entretanto, pode-se considerar que, em experimentação, dentro de certos limites, os custos, a não ser que sejam muito elevados, podem ser desprezados, com o objetivo de aumentar a precisão. No caso de experimentos com a cultura da mandioca, pelo menos com o nível tecnológico adotado na região, a utilização de parcelas com maior tamanho, dentro de certos limites, não levaria a grande aumento nos custos com a experimentação em campo. Tal afirmativa pode não ser verdadeira para outros tipos de experimentos.

Dentre os métodos utilizados neste trabalho, o método da máxima curvatura e comparação de variâncias estimam

Tabela 4. Estimativas de tamanho da parcela de mandioca $\left(\mathrm{m}^{2}\right)$ obtidas pelos métodos da máxima curvatura (MMC), máxima curvatura modificado (MMCM) e comparação de variâncias (MCV), Vitória da Conquista, UESB, 1998.

\begin{tabular}{lccc}
\hline \multicolumn{1}{c}{ Características } & MMC & MMCM & MCV \\
\hline Produção de raízes tuberosas & 28,80 & 24,14 & 28,80 \\
Peso da parte aérea & 28,80 & 26,59 & 28,80 \\
Índice de colheita & 14,40 & 18,00 & 14,40 \\
Porcentagem de matéria seca & 14,40 & 8,69 & 28,80 \\
Porcentagem de amido & 14,40 & 9,50 & 28,80 \\
Altura da planta & 14,40 & 15,89 & 28,80 \\
\hline
\end{tabular}

parcelas que coincidem com o tamanho das unidades básicas predeterminadas, isto é, não admitem encontrar valores intermediários entre as unidades básicas, a não ser que, no caso do primeiro método, o ponto de máxima curvatura seja determinado numa curva livre, com pouca precisão, e não por segmentos de reta, como adotou-se neste trabalho. Pela Tabela 5, observa-se que esses dois métodos estimaram parcelas com dimensões que variaram de $14,40 \mathrm{~m}^{2}$ a $28,80 \mathrm{~m}^{2}$, para as diversas características avaliadas, enquanto o método da máxima curvatura modificado estimou parcelas que variaram de $8,69 \mathrm{~m}^{2}$ a $26,59 \mathrm{~m}^{2}$.

Deste modo, o método da máxima curvatura modificado, tem a vantagem de estabelecer uma equação de regressão que normalmente apresenta altos valores de coeficiente de determinação, para encontrar o tamanho adequado de parcela, aumentando a confiabilidade das estimativas e permitindo encontrar valores intermediários entre os tamanhos de parcela pré-determinados no experimento, sendo por isto considerado o mais adequado.

Em experimentos com mandioca, geralmente as características aqui consideradas são analisadas, sendo portanto necessário escolher uma parcela que permita a adequada avaliação de todas elas. Assim, o tamanho adequado de parcela deve ser de $26,59 \mathrm{~m}^{2}$, com aproximadamente 44 plantas úteis, tomando-se como base a avaliação de peso da parte aérea, que exigiu parcelas de maior tamanho.

As informações sobre tamanho adequado de parcela em experimentos com mandioca são escassas e variáveis, principalmente considerando-se trabalhos realizados no Brasil e, ou, em regiões semi-áridas. As estimativas do tamanho das parcelas aqui obtidas (Tabela 4) estão próximas dos valores encontrados na literatura: 5 a 80 m² (Monzón et al., 1977, citados por Tineo \& Villasmil ,1988), $50 \mathrm{~m}^{2}$ (Sinthuprama et al., 1973) e 9,6 $\mathrm{m}^{2}$ (Bueno \& Gomes, 1983).

Pode-se concluir que as estimativas do tamanho da parcela variaram com os métodos empregados e com a característica analisada. O método da máxima curvatura e o método da comparação de variâncias estimaram parcelas com dimensões às vezes semelhantes, para as diversas características avaliadas, geralmente apresentando maior tamanho do que as parcelas estimadas pelo método da máxima curvatura modificado.

O método da máxima curvatura modificado permitiu a obtenção de estimativas mais adequadas, cujas dimensões mínimas variaram de $8,69 \mathrm{~m}^{2}$, para avaliação de porcentagem de matéria seca de raízes tuberosas, e cujas dimensões máximas foram de $26,59 \mathrm{~m}^{2}$, para avaliação de peso da parte aérea.

Para a eficiente avaliação de todas as características analisadas neste experimento, parcelas de $26,59 \mathrm{~m}^{2}$ (44 plantas) estimadas pelo método da máxima curvatura modificado, foram as mais adequadas.

\section{LITERATURA CITADA}

BUENO, A.; GOMES, F.P. Estimativa do tamanho de parcela em experimento de mandioca. $R e$ vista Brasileira de Mandioca, Cruz das Almas, v. 2, n. 2, p. 39-44, 1983.

CHAVES, L.J. Tamanho da parcela para seleção de progênies de milho (Zea mays L.). Piracicaba: ESALQ, 1985, 148 p. (Tese. Doutorado).

DOMINGUEZ, C.; CEBALLOS, L.F.; FUENTES, C. Morfologia de la planta de yuca. In: Yuca: Investigation, production y utilization. Cali, Colombia:CIAT/PNUD., 1982. p. 28-49, 1982. 
FAO. Faostat database gateway. 2000. http:// apps.fao.org/lim500/nph-wrap.pl? Production. Crops.Primary\&Domain=SU. Consultado em 25 de jul. de 2000.

FEDERER, W. T. Experimental design. New York: MacMillan, Company. 1955. 544 p. GROSSMANN, J.; FREITAS, A.C. Determinação do teor de matéria seca pelo peso específico em raízes de mandioca. Revista Agronômica, Porto Alegre, v. 160/162, n. 4, p. 75-80, 1950.

HATHEWAY, W.H.; WILLIAMS, E.J. Efficient estimation of the relationship between plot size and the variability of crop yields. Biometrics, v. 14, p. 207-222, 1958.

LESSMAN, K.J.; ATKINS, R.E. Optimum plot size and relative efficiency of lattice designs for grain sorghum yield tests. Crop Science, v. 3, n. 5 , p. 477-481, 1963.

LIN, C.S.; MORRISON, M.J.; BINNS, M.R. Persistence of a field heterogeneity index. Canadian Journal of Plant Science, v. 76, p. 245250, 1996.
LOZANO, J.C.; TORO, J.C.; CASTRO, A.; BELLOTI, A.C. Produção de material de plantio de mandioca. Cali, Colombia: CENTRO INTERNACIONAL DE AGRICULTURA TROPICAL. 28 p. 1977. (Série GP, 17).

MEIER, V.D.; LESSMAN, K.J. Estimation of optimum field plot shape and size for testing yield in Crambe abyssinica Hochst. Crop Science, v. 11, p. 648-650, 1971.

OLIVEIRA, P.H.; ESTEFANEL, V. Tamanho e forma da parcela para avaliação do rendimento em experimentos com batata. Ciência Rural, Santa Maria, v. 25, p. 205-220, 1995.

ORTIZ, R. Plot techniques for assessment of bunch weight in banana trials under two systems of crop management. Agronomy Journal, v. 87, p. 63-69, 1995.

ROCA, W.M.; NOLT, B.; MAFLA, G.; ROA, J., REYES, R. Eliminación de virus e propagación de clones en la yuca ( $M$. esculenta Crantz). In: ROCA, W.M.; MROGINSKI, L.A. (Eds.) Cultivo de tejidos en la agricultura, fundamentos $y$ aplicaciones. Cali, Colombia: CENTRO INTERNACIONAL DE AGRICULTURA TROPICAL. 1991. p. 403-420.
SINTHUPRAMA, S.; THIRAPORN, C.; SONHLAKSAP, N. Study on plot size and plot shape for cassava experiments. Progress report of 1973, Thailand Department of Agriculture. p. 262269.

STEEL, R.G.D.; TORRIE, J.H. Principles and procedures of statistics. 2a ed. New York: McGraw-Hill Book,. 1980. 633 p.

TINEO, J.R.; VILLASMIL, J.J. Determinacion del tamano optimo de parcela experimental en yuca (Manihot esculenta Crantz). Revista de la Facultad de Agronomia, Venezuela, v. 7, n. 2, p. 116-126, 1988

VALLEJO, R.L.; MENDOZA, H.A. Plot technique studies on sweet potato yield trials. Journal of the American Society for Horticultural Science, v. 117, n. 3, p. 508-511, 1992.

FREIRE JÚNIOR, M.; DELIZA, R.; CHITARRA, A.B. Alterações sensoriais em alface hidropônica cv. Regina minimamente processada e armazenada sob refrigeração. Horticultura Brasileira, Brasília, v. 20, n. 1, p. 63-66, março 2.002.

\title{
Alterações sensoriais em alface hidropônica cv. Regina minimamente processada e armazenada sob refrigeração.
}

\author{
Murillo Freire Júnior ${ }^{1}$; Rosires Deliza ${ }^{1}$; Adimilson Bosco Chitarra ${ }^{2}$ \\ ${ }^{1}$ Embrapa Agroindústria de Alimentos, Av. das Américas, 29501, Guaratiba, 23.020-470 Rio de Janeiro-RJ. E-mail: \\ mfreire@ctaa.embrapa.br; ${ }^{2}$ UFLA, Caixa Postal 37, CEP 37.200-000 Lavras-MG
}

\section{RESUMO}

Com o aumento da demanda por frutas e hortaliças minimamente processadas, a manutenção da qualidade destes produtos é um fator fundamental. $\mathrm{O}$ consumidor exige um produto fresco, de aspecto saudável, com boas características de cor, bem como outros atributos desejáveis na sua aparência. Foram avaliadas algumas alterações sensoriais em alface hidropônica, cv. Regina, minimamente processada, acondicionada em sacos plásticos termosoldados e armazenadas a $2^{\circ} \mathrm{C}$ e $10^{\circ} \mathrm{C}$. Independente da temperatura de armazenamento, até o terceiro dia, nenhum comprometimento do produto houve para qualquer dos aspectos de qualidade analisados: cor, frescor, escurecimento da nervura central, escurecimento de bordas, aspecto de podre e sabor de cozido. No armazenamento a $2^{\circ} \mathrm{C}$ a alface apresentou uma vida de prateleira de sete dias, sem comprometimento das suas principais características de aparência, ou seja, não houve escurecimento e a cor e o frescor se mantiveram inalterados. Para o produto armazenado a $10^{\circ} \mathrm{C}$, as alterações ocorridas tornaram o produto indesejável após o terceiro dia.

\section{ABSTRACT}

Sensorial changes of minimally processed hydroponic lettuce cv. Regina stored under refrigeration.

The maintenance of the sensorial quality of minimally processed fruits and vegetables is becoming a fundamental factor due to the increasing demand for these products. The consumer looks for a fresh product with healthy appearance and good color characteristics, as well as other desirable attributes in its appearance. Some sensorial characteristics were evaluated in minimally processed hydroponic lettuce, cv. Regina conditioned in thermally sealed plastic bags and stored at $2^{\circ} \mathrm{C}$ and $10^{\circ} \mathrm{C}$. For both storage temperatures, there was no significant product modification regarding the analyzed quality aspects like color, freshness, browning, rotten aspect and cooked flavor up to the third day of the experiment. At $2^{\circ} \mathrm{C}$ the lettuce presented a shelf life of 7 days without compromising its main sensorial characteristics as color, freshness and browning. For the product stored at $10^{\circ} \mathrm{C}$ the shelf life was up to 3 days.

Keywords: Lactuca sativa, fresh-cut, shelf life.

Palavras-chave: Lactuca sativa, pré-cortados, conservação.

\section{(Aceito para publicação em 03 de dezembro de 2.001)}

$\mathrm{O}$ consumidor vem apresentando cada vez maior consciência na escolha de sua alimentação, porém com menor tempo disponível para preparar refeições saudáveis. Como resultado, o mercado e a demanda por frutas e vegetais minimamente processados têm aumentado rapidamente, proporcionando o surgimento de produtos convenientes, ou seja, produtos frescos que podem ser preparados e consumidos em pouco tempo (Burns, 1995). Seguindo uma tendência mundial, muitos consumidores brasileiros resolveram utilizar pratos semi-prontos e vegetais pré-cortados. Os 